\title{
Iatrogenic Pneumothorax During Parathyroid Gland Biopsy: A Case Report
}

\author{
İsmail Ertuğrul GEDİK ${ }^{\mathrm{a}^{*}}$ \\ ${ }^{a}$ Erzurum Regional Education and Research Hospital Thoracic Surgery Department, 25000, Erzurum, TURKEY.
}

\begin{abstract}
An important subgroup of traumatic pneumothoraces, iatrogenic pneumothorax may occur during a variety of invasive procedures, including surgeries. In this report, we present a case of iatrogenic pneumothorax that occurred during parathyroid gland biopsy. A 54-year-old male patient arrived at our clinic complaining of acute onset right-sided chest pain and dyspnea that occurred during parathyroid gland biopsy. The patient's physical examination revealed diminished respiratory sounds in the right hemithorax. Pneumothorax in the right hemithorax. Chest tube thoracostomy was immediately performed through the right fifth intercostal space. While air drainage ceased on the second post-procedure day, auscultation revealed diminished respiratory sounds in the right apical zone on the fourth post-procedure day. Chest x-ray was performed on the same day, revealing lung expansion defect. The chest tube was clamped and a chest x-ray was repeated two hours later, which showed no significant difference. The chest tube was removed. Control physical examination and chest x-ray did not reveal any significant abnormalities and the patient was discharged. It should always be kept in mind that perithoracic and cervical invasive procedures may cause iatrogenic pneumothorax. Keywords: Pneumothorax; Thoracostomy; Biopsy; Fine-Needle; Iatrogenic Disease
\end{abstract}

\section{INTRODUCTION}

Pneumothorax is the pathological accumulation of air in the pleural cavity. Pneumothoraces can be spontaneous or traumatic. Iatrogenic pneumothorax (IPx) is an important subgroup of traumatic pneumothoraces. IPx may occur during many invasive procedures, including surgeries ${ }^{[1]}$. Herein, we present a case of IPx resulting from parathyroid gland biopsy.

\section{CASE REPORT}

A 54-year-old male patient was consulted to our clinic with complaints of acute onset right-sided chest pain and dyspnea that occurred during parathyroid gland biopsy performed by the endocrinology clinic. The patient's physical examination findings were as follows:

\footnotetext{
*Corresponding author: İsmail Ertuğrul GEDiK

Mailing address: Erzurum Regional Education and Research Hospital Thoracic Surgery Department, 25000, Erzurum, TURKEY.

E-mail: ertugrulgedik@gmail.com

Received: 30 December 2019 Accepted: 25 February 2020
}

blood pressure of 130/88 $\mathrm{mm} \mathrm{Hg}$, heart rate of 96/min, and respiratory rate of $26 / \mathrm{min}$. Lung auscultation revealed diminished respiratory sounds in the right hemithorax. Posterior-anterior (PA) chest x-ray revealed a pneumothorax in the right hemithorax (Figure 1). Chest tube thoracostomy using a 28 French (Fr) drain was performed immediately through the intersection of the right fifth intercostal space and mid-axillary line. Air drainage stopped on the second post-procedure day, yet auscultation revealed diminished respiratory sounds in the right apical zone on the fourth post-procedure day. PA chest $x$-ray was performed on the same day, revealing lung expansion defect (Figure 2). The chest tube was clamped and PA chest x-ray was repeated 2 hours later, showing no significant difference. The chest tube was removed. Control physical examination and PA chest x-ray did not reveal any significant abnormalities. The patient was discharged and invited for a follow-up outpatient clinic examination.

\section{DISCUSSION}

Anatomy of the apical part of the parietal pleura is important in understanding the etiopathophysiology of IPx. The upper 


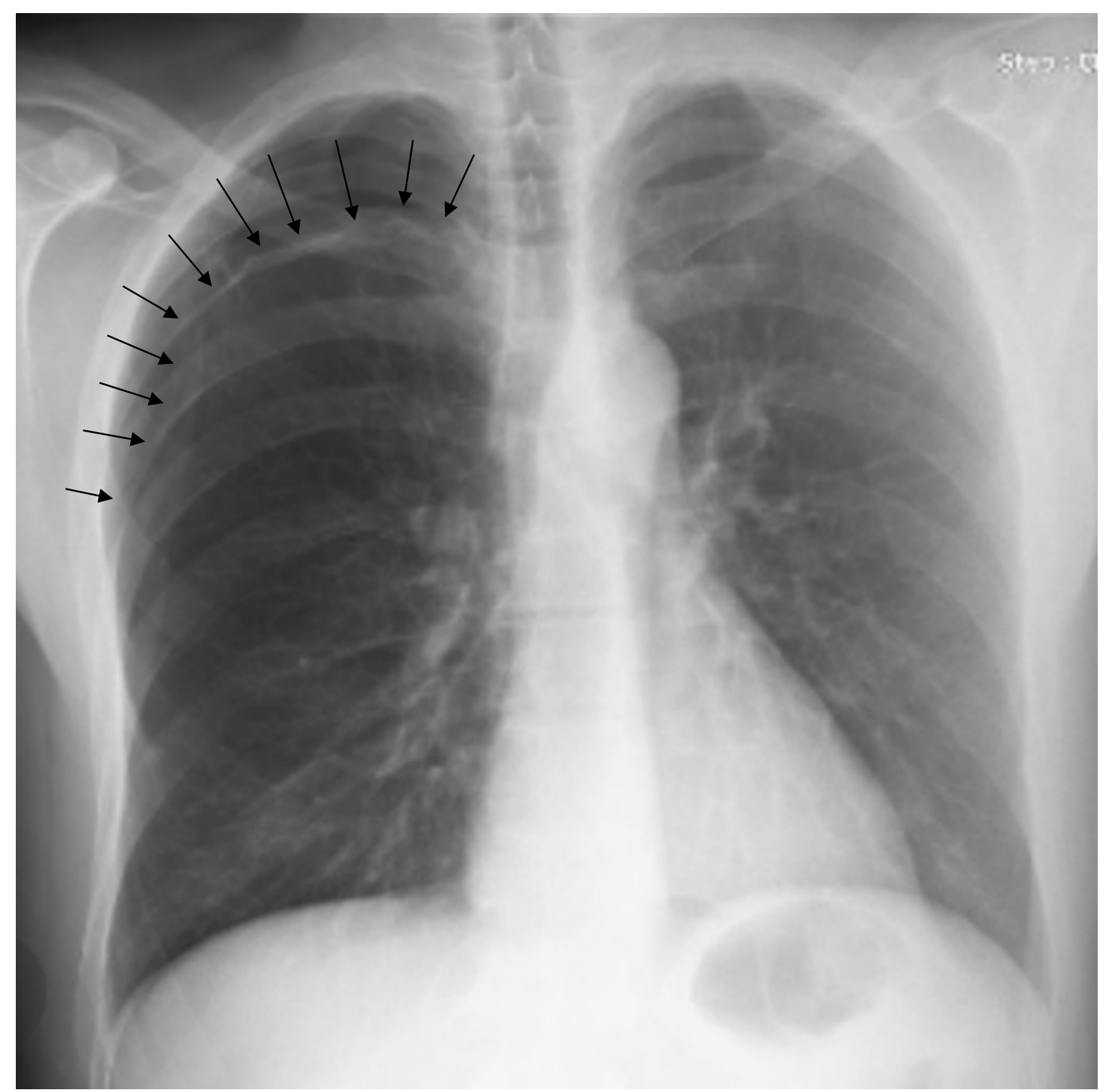

Figure 1. Right-sided pneumothorax on PA chest x-ray.

part of the pleura and pulmonary apex occupy the thoracic inlet on both sides. Between the pleura and neck of the first rib are the superior intercostal artery, as well as the sympathetic trunk and ventral ramus of the first thoracic nerve. The internal thoracic artery enters the thorax between the first costal cartilage and pleura ${ }^{[1]}$.

The most common cause of IPx is subclavian catheter insertion. Transthoracic/transbronchial fine needle biopsy and positive pressure mechanical ventilation are also among the top etiological procedures for IPx ${ }^{[2]}$. Additionally, IPx has been reported to occur during axillary lymph node biopsy, hypoglossal nerve stimulation, and acupuncture ${ }^{[3-5]}$. No case report of IPx occurrence during parathyroid gland biopsy was found.
IPx incidence is reported to be $1.36 \%$, increasing with the number of invasive interventions ${ }^{[6]}$.

The pathophysiology of IPx is similar to non-iatrogenic traumatic pneumothoraces. In IPx, the visceral pleura is usually damaged, possibly leading to life-threatening tension pneumothorax. Thus, symptoms and signs include decreased or absent breath sounds on the affected side, pleuritic pain and dyspnea, tachypnea, and tachycardia. If tension pneumothorax is present, hypotension, bradycardia, and cardiopulmonary arrest may occur ${ }^{[7]}$.

The diagnosis of IPx is also similar to non-iatrogenic types. In conjunction with physical examination findings, radiological methods such as PA chest $\mathrm{x}$-ray, thoracic computerized tomography, and thoracic ultrasonography may help the phy- 


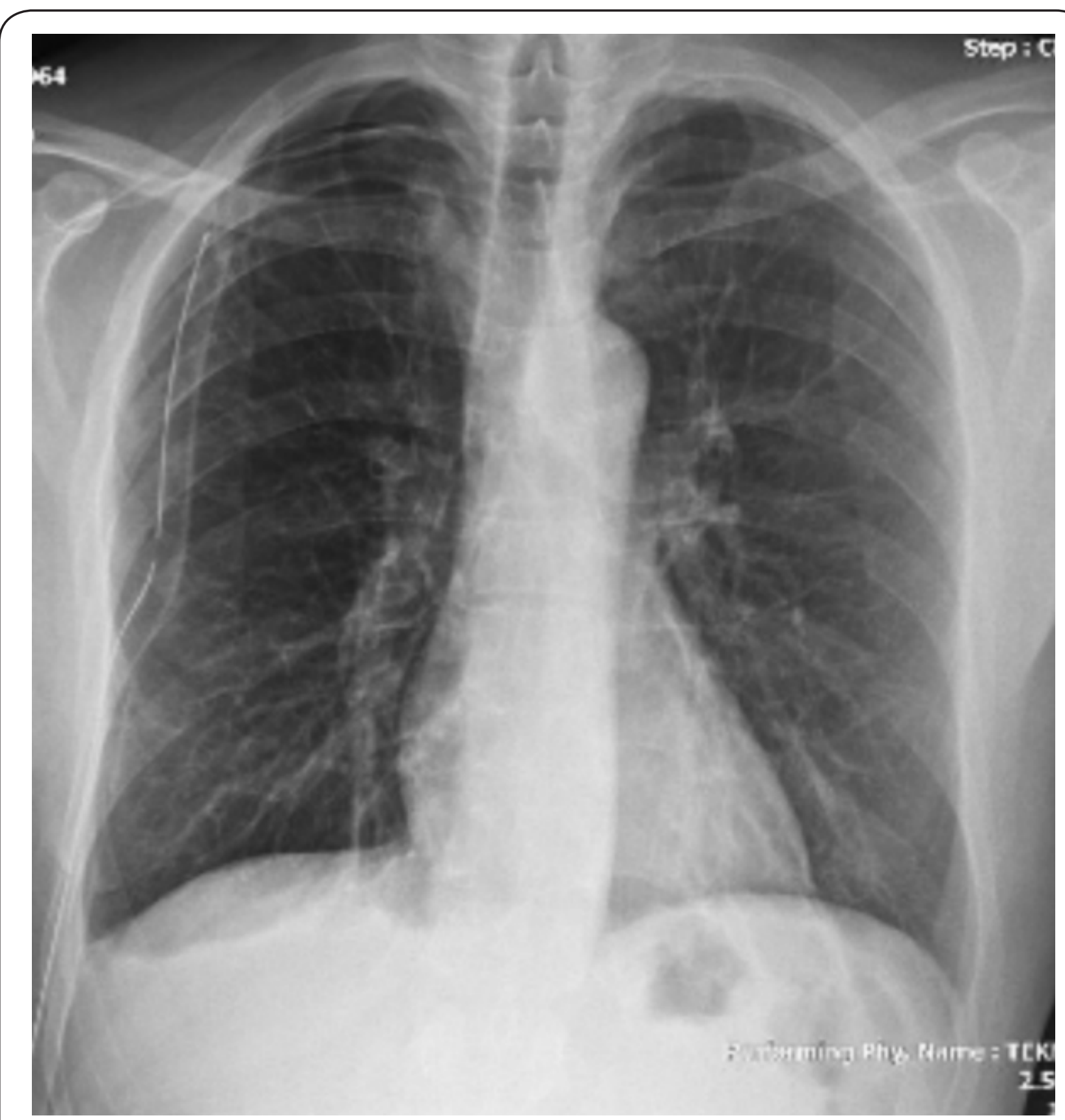

Figure 2. Right lung expansion defect seen on PA chest x-ray at fourth post-procedure day.

sician in diagnosing $\operatorname{IPx}^{[7]}$.

There are several methods to calculate the amount of pneumothorax using radiological findings. We use Light index in our clinic for this calculation. In the Light index, the pneumothorax size is a function of the average diameter at the midpoint of the cranial half of the hemithorax (y) and the average diameter at the midpoint of the cranial half of the lung (x). Thus, the Light index is calculated as Light $\%=100$ $-100 x(x / y)^{3}{ }^{[8]}$.

Treatment of IPx is similar to any other type of pneumothorax and is dependent on severity. Simple observation with oxygen insufflation can be curative in cases of mild pneumothoraces ( $<20 \%$ of the volume of the invovled hemithorax) as long as the patient is stable. Fine needle aspiration can also be considered as a treatment. Chest tube thoracostomy is the treatment of choice in large volume pneumothoraces and unstable cases ${ }^{[7]}$. In conclusion, it should always be kept in mind that perithoracic invasive procedures may cause IPx. If IPx is suspected, thoracic surgery clinics should be consulted immediately, thereby preventing potential morbidity and mortality attributable to pneumothorax.

\section{DECLARATIONS}




\section{Authors' contributions}

Made substantial contributions to conception and design of the study and performed data analysis and interpretation. Performed data acquisition, as well as provided administrative, technical, and material support: Gedik İ E.

\section{Conflicts of interest}

All authors declared that there are no conflicts of interest.

\section{REFERENCES}

1. Charalampidis, C., Youroukou, A., Lazaridis, G., Baka, S., Mpoukovinas, I., Karavasilis, V., ... \& Tsakiridis, K. (2015). Pleura space anatomy. Journal of thoracic disease, 7(Suppl 1), S27.

2. Ojeda, J. R., \& Hipskind, J. E. (2018). Pneumothorax, Iatrogenic.

3. Stefani, A., Ruggiero, C., Aramini, B., \& Scamporlino, A. (2018). An unusual drain in the pleural cavity: iatrogenic pneumothorax due to pulmonary misplacement of a nasogastric tube. Intensive care medicine, 44(12), 22902291.
4. Arteaga, A. A., Pitts, K. D., \& Lewis, A. F. (2018). Iatrogenic pneumothorax during hypoglossal nerve stimulator implantation. American journal of otolaryngology, 39(5), 636-638.

5. Tagami, R., Moriya, T., Kinoshita, K., \& Tanjoh, K. (2013). Bilateral tension pneumothorax related to acupuncture. Acupuncture in Medicine, 31(2), 242-244.

6. Celik, B., Sahin, E., Nadir, A., \& Kaptanoglu, M. (2009). Iatrogenic pneumothorax: etiology, incidence and risk factors. The Thoracic and cardiovascular surgeon, 57(05), 286-290.

7. Loiselle, A., Parish, J. M., Wilkens, J. A., \& Jaroszewski, D. E. (2013). Managing iatrogenic pneumothorax and chest tubes. Journal of hospital medicine, 8(7), 402-408.

8. Salazar, A. J., Aguirre, D. A., Ocampo, J., Camacho, J. C., \& Díaz, X. A. (2014). Evaluation of three pneumothorax size quantification methods on digitized chest X-ray films using medical-grade grayscale and consumergrade color displays. Journal of digital imaging, 27(2), 280-286. 